\title{
La Suisse rejoint l'Alliance Européenne ESTHER - quelles perspectives?
}

\author{
Nathalie Mezger, \\ Thomas Vogel, Beat Stoll, \\ Alexandra Calmy
}

Correspondance: Dr Nathalie Mezger, MD, DTMH, MPH

Cheffe de Clinique Hôpitaux Universitaires de Genève (HUG) Service de Médecine Internationale et Humanitaire (SMIH) Av. Gabrielle-Perret-Gentil 6, CH-1211 Genève 14 Tél. 0223729658 nathalie.mezger[at]hcuge.ch
Le 16 novembre 2011, la Suisse, via le Département fédéral des affaires étrangères (DFAE) et la Direction du développement et de la coopération (DDC), a décidé de rejoindre les dix autres pays membres de l'Alliance européenne ESTHER. Il s'agit de l'aboutissement d'une longue réflexion, mais surtout à présent, de la genèse d'une initiative nationale, car désormais, l'entité ESTHER Suisse doit croître et s'affirmer comme un pilier de la solidarité suisse.

\section{Mais qu'est-ce donc que l'Alliance ESTHER?}

L'Alliance Européenne ESTHER, Ensemble pour une Solidarité Thérapeutique Hospitalière en Réseau, est une initiative française à l'origine. Depuis 2002, elle fédère un nombre croissant de pays autour d'une charte et de principes de base dans la lutte contre le VIH/SIDA et ses conséquences dans les pays en développement ou en transition (pour plus de détails voir www.esther.eu).

L'originalité de cette initiative tient au fait que les coopérations soutenues par les pays membres s'expriment par la création de partenariats hospitaliers Nord-Sud, mais aussi Sud-Sud.

L'Alliance ESTHER se base sur le développement conjoint d'un véritable réseau de personnels de santé visant à développer des capacités locales en réponse aux besoins identifiés par les partenaires sur place. Il s'agit avant tout de favoriser l'accès aux soins, d'en améliorer leur qualité, mais en aucun cas de développer des secteurs hospitaliers sophistiqués, éloignés des communautés...

Les hôpitaux, partie intégrante des systèmes de santé, jouent un rôle essentiel tant pour la fourniture de soins que pour la formation, la détection, la prévention ou encore la recherche (OMS 1987). A ce titre, ils ne peuvent plus être écartés d'une politique de coopération raisonnée, visant des résultats à long terme sur la santé des populations. De leur côté, les hôpitaux ne peuvent désormais plus ignorer les autres acteurs du système de santé. Complémentaires, ils se doivent de collaborer autour du patient, au sein de sa communauté, avec l'ensemble des prestataires de soins.

Continuité et échanges dans le système de santé sont au cœur des partenariats soutenus par l'Alliance ESTHER, c'est pourquoi les projets initiés incluent souvent, en sus des hôpitaux, la société civile et différents groupes extrahospitaliers. Ses activités sont donc fréquemment réalisées hors du cadre hospitalier.

\section{Résumé}

Le 16 novembre 2011 la Suisse a rejoint officiellement les dix autres pays membres de l'alliance européenne ESTHER, dite Ensemble pour une Solidarité Thérapeutique Hospitalière en Réseau. Cette alliance fédère, au travers de partenariats, pays et hôpitaux dans la lutte contre le VIH/SIDA et ses conséquences dans les pays en développement ou en transition. Après une brève présentation de l'alliance européenne ESTHER cet article décrit quelques défis actuels auxquels tant l'alliance que la Suisse vont devoir faire face, à savoir:

- Permettre une certaine flexibilité à ces collaborations alors qu'elles se basent sur des partenariats gouvernementaux et hospitaliers;

- Accepter l'élargissement de la thématique de base, le HIV/SIDA, parce que désormais certains de ses membres mettent l'accent sur les partenariats interhospitaliers et le développement de capacité locale;

- Créer une entité ESTHER Suisse la plus fonctionnelle possible.

Depuis ses débuts, plus de 120 programmes dans plus de 40 pays ont été créés. Ces programmes y déclinent des activités variées, telles que l'enseignement ou l'accompagnement de professionnels de la santé (médecin, infirmiers, biologistes, administrateurs, etc.), le développement de compétences, la fourniture d'assistance technique et/ou de la recherche clinique de terrain.

\section{Quels sont les défis qu'ESTHER devra relever?} Premier défi: élargir les thèmes des collaborations interhospitalières:

Si l'objectif fédérateur initial, le VIH/SIDA, peut paraître restrictif, il faut souligner qu'en réalité il permet déjà une approche thématique très large du fait que cette problématique est intimement liée à la qualité des soins dans son ensemble et requiert tant une expertise transversale, qu'une coordination 
de tous les prestataires de services de santé, hospitalière et/ou extrahospitalière. A titre d'exemple, on peut citer les projets touchant à la sécurité transfusionnelle, à l'hygiène hospitalière, à la gestion des déchets ou de la pharmacie, à la qualité des laboratoires, aux services aux populations vulnérables (mère/enfant, prisonniers, dépendants), à l'approvisionnement et à la gestion nationale des médicaments, à la souscription au Fonds mondial de lutte contre le sida, la tuberculose et le paludisme...

Bien que la lutte contre l'épidémie du VIH/SIDA y reste une pierre d'angle, avec le temps certains membres de l'Alliance ESTHER mettent désormais davantage l'accent sur le partenariat, le développement de capacité locale, l'amélioration du système de santé dans son ensemble et la réalisation des Objectifs du millénaire pour le développement (MDG 4, 5, 6)...
Simple en théorie, le travail à plusieurs, en partenariat, en réseau, est plus complexe dans les faits. Très enrichissant et constructif, il peut, si l'on n'y prend garde, devenir vite inefficace, voire inhibiteur. Ainsi, il sera capital de tenir compte des attentes, des particularités et des capacités de chacun des partenaires principaux, gouvernements et hôpitaux, et de toujours mettre le patient et la population bénéficiaire au centre du débat.

Les gouvernements, tant du Nord que du Sud, doivent être la force motrice de l'Alliance. Au travers de leur engagement, de leur appui politique et financier, ils peuvent viser une meilleure intégration, une pérennisation des projets et un impact durable pour les populations bénéficiaires.

L'Alliance de son côté, prendra en considération les organisations et les attentes des pays membres et des donateurs. Tout gouvernement a ses priorités et ses exigences en matière de fonctionnement et doit

\section{«ll faut faire de l'hôpital élargi un partenaire valorisé de la coopération au développement.»}

Cet élargissement thématique est le bienvenu. Il témoigne du potentiel de l'Alliance de s'inscrire dans une coopération médicale plus engagée et est un reflet de son dynamisme et de sa capacité à dialoguer Deuxième défi: travailler en collaboration, en partenariat, inclure davantage les gouvernements:

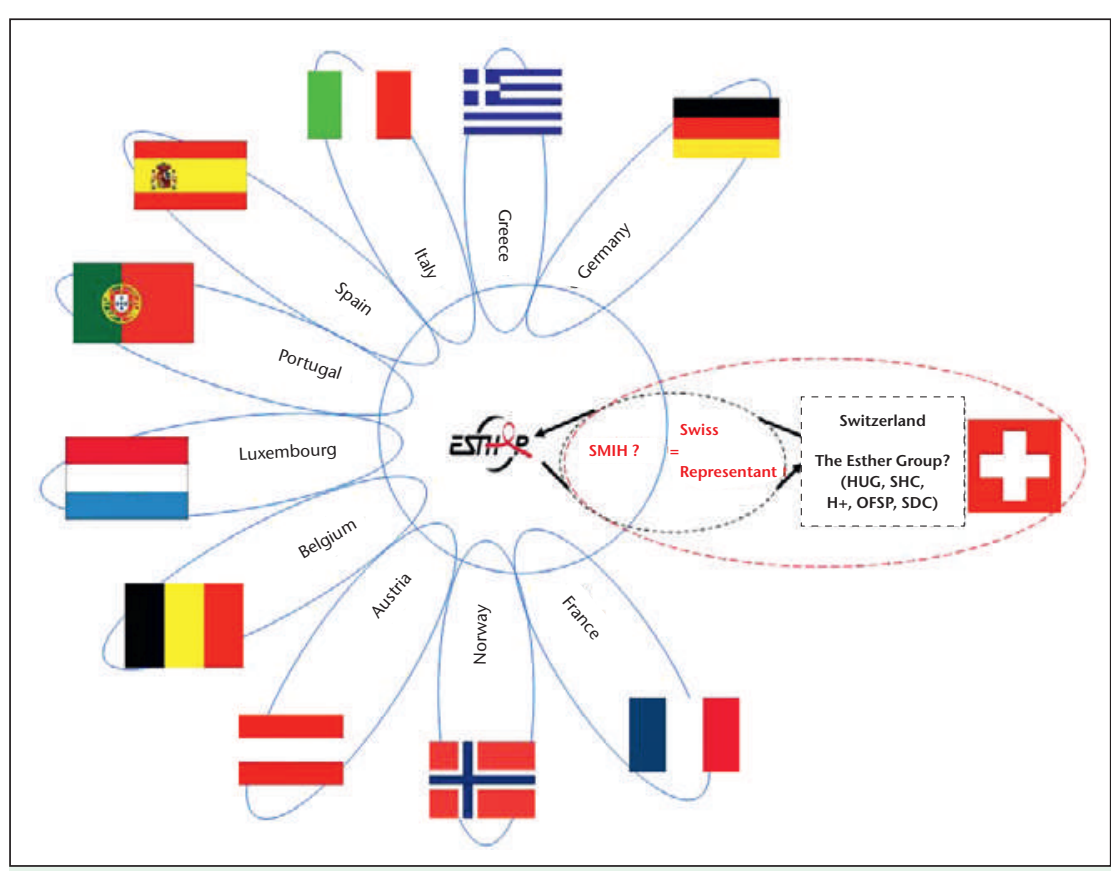

Le 16 novembre 2011 la Suisse a rejoint officiellement les dix autres pays membres de I'alliance européenne ESTHER, dite Ensemble pour une Solidarité Thérapeutique Hospitalière en Réseau. rendre compte à ses citoyens et ses résidents des résultats atteints avec ses deniers. L'Alliance devra permettre cette restitution.

Troisième défi: Faire de l'hôpital élargi un partenaire valorisé de la coopération au développement:

L'aspect innovant des partenariats ESTHER (base hospitalière, actions médicales) tombe parfois hors du cadre usuel de la coopération au développement. Par conséquent, si les gouvernements cautionnent la thématique et l'approche d'ESTHER, il devrait leur être possible de garder une certaine souplesse et de prendre en compte les particularités de cet autre partenaire principal qu'est l'institution hospitalière au sens large.

En effet, les hôpitaux, vivier d'expertise et d'expériences, sont dans ESTHER les forces vives et effectrices de l'Alliance. En Suisse, ceux-ci n'ont d'ailleurs pas attendu cette initiative pour réaliser des projets internationaux de développement en partenariats. Une enquête conduite par le Service de médecine internationale et humanitaire des Hôpitaux universitaires de Genève (SMIH/HUG) a révélé que 30 établissements totalisent 112 projets de coopération. Ces partenariats, non en réseau, débutent en général sur une base interpersonnelle, spontanée, une occasion, aboutissant à la réalisation d'un petit projet. La confiance s'installant, ils s'étoffent et mènent souvent au développement de multiples collaborations, multisectorielles. Leurs fruits ne sont toutefois souvent pas capitalisés, ni partagés, ni même valorisés. L'Alliance peut ainsi apporter une possibilité de partager les connaissances et pratiques de ces projets afin d'en retirer les meilleurs leçons. 


\section{ESTHER Suisse: une opportunité à saisir? Ou un terrain semé d'embûche?}

Si l'intérêt en Suisse de partager, d'échanger, d'aplanir certaines inégalités de notre monde est évident, tout ceci prend du temps, de l'énergie et des ressources. Ces projets actifs, bien réels, occupent passablement certaines équipes à un moment où les hôpitaux, voyant leurs ressources diminuer, doivent faire face à des pénuries de personnels et des difficultés économiques. N'oublions pas que l'objectif principal d'un hôpital - fut-il dans une «ville internationale» - n'est pas la coopération internationale, même si en Suisse, les Hôpitaux universitaires de Genève (HUG) ont inscrit la collaboration internationale dans leur plan stratégique.

Pour être pertinente et efficace, ESTHER Suisse devrait être une organisation construite de manière concertée, impliquant les entités étatiques ainsi que les hôpitaux intéressés. Dans une phase initiale, il conviendrait de privilégier une structure légère (par ex. la création d'une association, indépendante des différents partenaires) pour préserver son agilité et

\section{Une organisation, des rôles et des responsabilités à définir}

Quelque soit l'identité choisie et la forme adoptée, la création d'ESTHER Suisse comprenant les principaux partenaires intéressés (Gouvernement[s], hôpitaux) est souhaitable dès à présent. Précisons, qu'à terme, l'Alliance pourrait accueillir d'autres institutions, associations ou individus clés, impliqués ou simplement intéressés par les partenariats hospitaliers au bénéfice du développement.

Les rôles, responsabilités, objectifs et moyens devront être déterminés. La coordination et la représentation auprès de l'Alliance Européenne ESTHER pourraient être soutenus par la Confédération et être déléguées à un binôme composé d'un représentant de ses deux principaux acteurs, à savoir: le Gouvernement suisse et les hôpitaux suisses.

En conclusion, à l'heure où les fonds se raréfient, où des maladies sont artificiellement opposées et mises en compétition (chroniques transmissibles contre non-transmissibles), la création de synergismes, le partage de ressources, d'informations pour tenter de renforcer des systèmes de santé et des com-

\section{«Nous espérons que la Suisse aura l'ambition de développer davantage les partenariats hospitaliers Nord-Sud.»}

sa faculté de réagir rapidement à l'évolution du champ d'activité et des attentes tant des bailleurs de fonds que des partenaires des pays bénéficiaires.

A noter que selon les pays membres, ESTHER peut dépendre d'un ou plusieurs ministère(s). Ce panachage d'entités ministérielles, bien que plus lourd, permet parfois d'élargir le champ d'action de cette lutte et de surseoir à certaines restrictions propres à chacune (par ex.: logique de pays d'intervention pour certains contre logique de thématique pour d'autres). munautés locales en développant leur capacité à mieux intégrer différentes problématiques paraît tomber sous le sens. Car au bout de la chaîne, il n'y aura toujours qu'un seul et même patient, qu'une seule et même population, souffrant de plus en plus simultanément de multiples problèmes et devant recourir à un seul et même système de santé.

Nous espérons que la Suisse aura l'ambition de développer davantage les partenariats hospitaliers Nord-Sud et les intégrer pleinement aux objectifs d'une politique avisée de coopération au développement. 